\title{
Forensic Psychiatric Autopsy: A Challenge Assessment
}

\author{
Marques-Teixeira J * \\ Department of Forensic Psychiatry, University of Porto, \\ Portugal \\ *Corresponding author: Marques-Teixeira J , \\ Department of Forensic Psychiatry, University of Porto, \\ Portugal
}

Received: March 15, 2017; Accepted: April 28, 2017; Published: May 10, 2017

\section{Abstract}

The forensic psychiatric autopsy, although it is a relatively rare forensic practice, has particularities that make it an exercise in clinical psychiatry not only extremely interesting, but also extremely specific among the several practices in the field of civil law. More often, unilateral acts by deceased elders are being challenged by relatives based on the argument that agents would not have the willingness capacity when they did the act.

It is the responsibility of the forensic psychiatrist to define, in a retrospective manner and without access to direct examination of the agent-only through the information contained in the records or through other means of information-3 types of diagnoses of the agent in question at the time of the act: (1) nosological diagnosis; (2) a diagnosis of the degrees of freedom of being as a consequence of the former; (3) a diagnosis of the consequences on the willingness capacity from the previous two.

To illustrate this procedure, the author presents a summary of two reports of psychiatric autopsy, which were presented in court, ending with some considerations about this type of procedure.

Keywords: Forensic psychiatric autopsy; Forensic psychiatrist

\section{Introduction}

One of the activities for which the forensic psychiatrist is often called to intervene in civil law is to attest to a person's mental capacity at the time she caused an event that may be stipulated in civil law. The conceptual basis for this type of approach is based on the axiom that any forensic evaluation, including psychiatric evaluation, cannot be confused with a diagnosis because it represents a scientific report that provides the Justice System with a dynamic interpretation of a complex situation. In forensic psychiatry both psychiatric ability and mental fitness are based on the state of consciousness at the time of the act, but this state is not sufficient for the assessment of mental capacity as a whole. Therefore, in civil law, this capacity is defined as the mental capacity of a person to understand the content and especially the consequences of a unilateral (testament) or bilateral civil document (contracts, etc.). For this are considered either the state of normality or mental abnormality, or particular and understandable transitory states.

Most of the European states have laws that restrict a testator's right to disinherit a presumptive heir. In addition, the courts are very willing to distribute assets to close relatives, so this legal bias favors presumptive heirs, who are usually the ones who most contest a will or a disposition. However, consistently with the policies of the European Union [1], the Portuguese State has determined that an innovative course should be drawn in the sense that the fact that a person suffers from an illness that limits her mental and physical faculties does not mean, nor must determine that she is, therefore, legally prevented from exercising all the rights he holds, but the extent of the disability must be fixed on a case-by-case basis, depending on the circumstances.

When the assessment of those circumstances is made retrospectively, upon the agent's death, it is referred to as a psychiatric autopsy, in which the mental capacity is assessed retrospectively through the reconstruction of the agent's mental state at the moment of the act.

A special situation of the psychiatric autopsy is the situation in which the annulment of a testament of a deceased agent is required. Most of these will contests are based on whether the testator himself lacked capacity or was under undue influence when executing the will. What is in issue is to demonstrate that the testator had the mental capacity to execute the will. This involves a full and accurate assessment of the clinical or other documents in the file so that a decision can be reached on the agent's ability to decide at the time of the act under review. The psychiatric issues under discussion in these cases include the nosological classification, the symptoms that can be reconstructed from the documents available in the case, the reconstruction of the agent's motivational system, and, finally, the association of all this, as underlying patterns of decision. For people with a pathology requiring a clinical evaluation by an expert, both the comprehensibility of the motivation and the absence of manipulation due to volitional deficit must be demonstrated.

The retrospective diagnosis of a particular clinical situation, when the patient has already died and therefore we have not means to collect a direct previous clinical history or a direct clinical examination, the retrospective diagnosis is only possible if we have enough documents to do so. Even if we can obtain a clinical diagnosis, in order to appreciate the influence of this diagnosis on the willingness capacity of the agent to want and understand the scope of a given act, we would have to have in those documents an appreciation of willingness capacity on the part of the clinicians that have seen the agent close to act and the reason is simple: the direct correlation between a clinical diagnosis and the willingness capacity, to want and to understand 
the scope of a given act, simply does not exist or is very difficult to formulate, besides being variable between diagnoses and, within the same diagnosis, at different times. For example, in an earlier publication we refer to the following as regards schizophrenia (here, as an example only of the attribution of a diagnosis): "No one calls into question that an acute episode of schizophrenia or a condition typical of a schizophrenic defect imputability. This is mainly due to the fact that in these states the psychotic patient, at the moment of the act, is not able to assess the illicitness of the same or, even if she is, she is not be able to self-determine in function of that evaluation. But as the Bonn study and the subsequent investigations of nearly 900 schizophrenic patients have shown that there are frequent uncharacteristic basic states in which patients experience the basic symptoms as deficiencies, being able to adapt and compensate them, preserving the capacity of insight and reality testing. This means that the capacity to work and social life may be altered, while maintaining the responsibility and the civil capacity" [2]. That is, it is not the fact of having a diagnosis of schizophrenia that in itself makes its bearer a person with deficits in the ability to judge and, in this sense, to maintain the civil capacity and this is taken intuitively: mental and civil capacity is underpinned by considerations of autonomy, broadly conceived. It is beyond the scope of this article to offer a discussion on the many facets of autonomy and its relation with forensic psychiatric assessment [3-5]. The point to emphasize is that it was based on the relationships between autonomy and forensic psychiatric assessment that this clinical approach was developed with the intention of providing the court with the clinical-scientific bases of the relationship between mental illness and mental capacity, where the court decisions could be rooted.

To illustrate this approach we present two cases of psychiatric autopsy of our clinic, were the demand was to analyze if the deceived agent was having his willingness capacity preserved at the moment of making his unilateral document. These two cases were based only on the clinical material present in the forensic file, and were triggered by the family that contested de civil document.

\section{Case Presentation}

\section{Case 1}

This is a male that died in October 2010, suffering from an oncological disease. From the clinical observation reported in the clinical file at $4 / 17 / 2008$ (where, in addition to the references to oncological disease in progress, it is mentioned, with interest for this assessment, that the patient had no "pathological personal antecedents worthy of registration" or family members with psychiatric disorders), until the subsequent consultations referred to here to 7-1-10, there was never mentioned in the records of changes of a psychic nature, but rather of the physical symptoms resulting from the oncological treatments applied; even the prescription of antidepressants is part of the legis artis in these cases, in which it is recommended to take antidepressants to treat some depressive symptoms reactive to the oncological situation, understanding that the improvement of mood improves the acceptance of oncological, invasive and uncomfortable treatments.

On 04-29-10, his doctor reported the clinical evolution of the patient regarding his oncological condition, from 04-17-08 to 04-2110 , and at no time having referred to any change in the psychic state of the patient. This can even be verified by clinical records in the period between the dates mentioned above, by frequent references to "wellbeing" or "well-disposed patient". On 06-01-10 he is hospitalized due to febrile condition after a chemotherapy cycle, having been improved on 06-09-10. In the course of hospitalization, there are numerous references in the nursing records to the mental state of the patient who, after a short period of mental confusion (at the beginning of the hospital stay), quickly recovers and becomes "aware and oriented" until discharge.

On 06-22-10, he went to the hospital for an acute abdominal condition and was treated and improved on 06-23-10, without reference to any change in his psychic state.

On 06-30-10, the patient is observed by his usual doctor who declares that the patient "maintains his intellectual faculties".

At 07-30-10 he signs informed consent for cataract surgery, with a similar signature to the one used in informed consent dated of $05-$ 02-08 and 02-04-10.

On 08-18-10, about 1.5 months after the testament, the patient is again observed on Onco-Hematology, presented a good general condition, translated into an adequate weight $(62 \mathrm{~kg})$, and a general physical examination without changes registered, being to registered the fact that the clinician had to register in the clinical process the place where it was living ("Already living at your own house!"), indicating a marked improvement in its general condition.

The patient dies on 10-08-10.

Analysis of historiographical-clinical data: The patient in question suffered from cancer disease for which he underwent specific treatments started in 2008. Throughout the treatments the patient suffered from the usual side effects in this type of treatment (loss of strength, loss of appetite, nausea and vomiting, and reactive depression both to disease and to treatments). But he always recovers, to the point that he has returned to his usual residence.

Even in more troubled periods of side effects or parasitic infections, the patient always recovers from more confusional states of consciousness associated with weakened general state and hydroelectrolytic imbalance.

Throughout this period of long treatments there has never been any reference to any psychiatric condition that could interfere, on an ongoing basis, with the state of consciousness not even the depression associated with the oncologic picture had any interference in the state of consciousness.

The patient makes the testament on 06-06-10, and the next day his regular physician observes him and declares that he keeps his intellectual abilities preserved about 1 month after the will, the patient is subject to cataract surgery, having signed an informed consent, the signature of which is similar to the one made in previous informed consent and, a month and a half after the will he is again observed by his usual doctor and his normal mental state is confirmed.

From this analysis we can conclude the following: based on the clinical records in the file, and except for a short period of 1 day during hospitalization for febrile complications after chemotherapy and with rapid recovery, no alteration of consciousness or confusional 
state of the patient has ever been reported, despite the severity of the disease and the severity of the treatments. There is also little reference to psychiatric treatments for severe disturbance of consciousness or confusional state with behavioral changes.

The patient makes a will and, on the following day, was evaluated by his habitual doctor who declares the maintenance of his intellectual capacities. In addition, he resumed his habitual residence and underwent cataract surgery, having also signed an informed consent that presupposes a capacity for understanding and judgment about the medical act to which he was to be subjected, as well as the ability to decide on the subject, as well as to be aware of the risks involved.

That is, in psychiatric terms and based on clinical records; we have no indicators that tell us that the patient did not have the psychological conditions to exercise his willingness capacity. On the contrary, the clinical records go in the opposite direction.

So, in the field of forensic psychiatry, the questions that arise here are: Is it possible to deduce the ability to judge, retrospectively, on the basis of clinical records alone? If so, what are the main indicators that should be valued in this analysis? And what are the main constraints?

Regarding the first question, it is obvious that even with a postfacto observation of the mental state of an individual by direct examination; it cannot be stated with absolute certainty that at the time of the act the ability to judge was fully preserved. Much less by the analysis of clinical records without direct examination. That is, we are always talking about judgments of presumption based on an important fact of human life: its historical-vital continuity. That is, by removing exceptional situations, our psychic life presents a continuity of meaning that allows an experienced observer to deduce whether or not there was a break in that continuity of meaning at the moment of fact. But these judgments of presumption are probabilistic judgments that can be covered with a high or low probability of being in accordance with reality. The degree of probability will depend on the analysis of previous and post-facto events, events of medicalpsychiatric nature, with behavioral translation, that allow to deduce if the continuity referred to above was broken or not. This presupposes, first of all, the type of clinical picture under study, its complications and, very importantly, its impact on the psychic life regarding interference with the willingness capacity. It is in this analysis that the opinion delivered here is based.

Concerning the second question, the important factors to be taken into account have to do with the clinical observation of the general and psychological state of the patient throughout the treatments, as well as the intercurrence of medical or psychiatric events that may directly interfere in the ability to judge. The third question is directly related to the fact that if there are no indicators that allow us to make that assessment, it is not possible to obtain elements to deduce a psychiatric-forensic judgment. However, we must bear in mind that the absence of some indicators may be a factor to value, as we will see ahead.

Having said that, let us return to the present case and analyze it against the background described above.

a) What can we say about the historical-vital continuity in relation to the case being evaluated and the act in question: the will? The data from the records indicate, with a high degree of probability, that this continuity of meaning was not lost, since the patient had, 6 days before the will, a clinical evaluation of discharge from the hospital that indicated that the patient was well fitted in the psychic domain, because otherwise there should be any reference to the change in his psychic state and his referral to psychiatry. Likewise, on the day following the will, the patient is observed by his habitual doctor who declares, precisely, the permanence of this psychic state. That is, with a high degree of probability, the patient maintained its historical-life continuity before, during and after the testamentary act. However, it is important to know whether there is a high probability of a rupture of the historical-vital continuity, if this continuity was compatible with the ability to judge. That is, if there is no change in the way he lives, if, even then, the patient would be able to make an aware decision concerning the testamentary act and its consequences? Not suffering from a psychiatric condition known from clinical records, with the exception of reactive depression which, on its own, hardly changes that ability, only if his physical illness had metastases to the brain and as a consequence could change in a way more or less durable, the ability to judge. However, at no point in the clinical history did this situation arise, nor was there a need to resort to any diagnostic auxiliary examination to mislead this possibility. Of course we are talking about doctors with high experience in oncology, in which this possibility occurs in many neoplastic diseases.

b) What, then, are the main indicators that allow us, with a high probability, to suggest that the patient was capable of an aware decision at the time of the will? First of all, by the presence, affirmatively, of several clinical records continued in time, of the patient being conscious, oriented and, generally, with a mental state suited to his circumstances. But also by the lack of referral to psychiatry for clinical observation for a state that could alter the patient's consciousness and behavior, as well as the absence of requests for diagnostic tests to assess the state of functioning of the brain, as discussed above. In addition, a return to normal life, as reported in the clinical records, is an indicator of balance that deserves consideration.

Conclusion: There are no records in the file that allow us to state objectively that at the time of the will the patient suffered from changes in the capacity to judge, to want and to understand the scope of his act.

In view of the foregoing considerations, we are of the opinion that at the time of the will the probability of the patient having the capacity for judgment to want and to understand the scope of his act would have been vastly superior of not being able to want and to understand the scope of the act.

\section{Case 2}

This is a male that died in June 2013 by a heart attack. In November 2004, the patient was considered as having no structural pathology of the brain (assessed by computerized axial tomography and validated by a neurologist), nor presented mental deterioration (assessed by Mini Mental State Examination-MMSE-and validated by a neuropsychologist), having had a short hospitalization period with the diagnosis of "undiagnosed disease"), according to a document signed by the Clinical Director of the Psychiatric Hospital where he was as inpatient. Although, a later document is presented where one of the physician of the hospital report that the patient suffers from paranoid delirious syndrome. It is also reported that the patient will 
have had a myocardial infarction 30 years earlier.

Between 2004 and 2008 there were no clinical records, and for the first time the reference to the dementia syndrome appeared in a registry of 09-10-08, by his habitual psychiatrist. One year later (May 5, 2009) the same doctor notes that there was a worsening of memory, in January 2010, the same doctor report that the patient is compensated, in March of the same year that is depressed and in September of the same year that is stable. Between September 2010 and March 2011 there are no records of consultations (deducting that the examinee will not have made medical consultations), and on March 10, 2011, the patient have made a will and on June 29, 2011 there was again reference to dementia. On October 10, 2012, a reference to the lack of autonomy in daily life activities is reported for the first time. In summary, this is the sequence of events which are relevant to the present case.

Analysis of historiographical-clinical data: Let us examine some details of these events. In 2004 (7 years prior of making the will) the patient was clinically evaluated (by a psychiatrist and a neurologist) and performs ancillary diagnostic tests (CAT scan and MMSE) and is considered not suffering from structural changes of the brain.

Between 2004-2008 there are no clinical records. In September 2008, a dementia syndrome register appeared without any reference to the degree, type and data that supported the diagnosis. That is, we do not know if the clinician only evaluated the patient by clinical interview method, if she applied or had him apply a set of obligatory neuropsychological tests, according to legis artis, to support a diagnosis of dementia syndrome. In the absence of reference to such in the file, we do not know the degree and the type of dementia syndrome, and if it exists at all.

The same happens 1 year later when the same doctor reported that there was a worsening of memory without specifying the degree of worsening and the type of memory that was aggravated was evaluated (if clinically, if instrumentally).

For about one year later, the same clinician reported that the patient was "compensated", a few months later "he was stable" and then "he was depressed", without specifying what she means by to be "compensated", "stable" or depressed: would compensated be equal to normal behavior, or normal functioning, or normal memory? And stable, would it be equal to normal mental functioning? And would depression be the same as having a diagnosis of depression or just having an increased sadness?

This is followed by a new period in which the patient has no medical records (between September 2010 and June 2011) during which the patient makes a will.

In June 2011 there is again reference to dementia, without specification or clarification and in October 2012 (more than 1 year later) there is a reference to the lack of autonomy in activities of daily living.

From this analysis we can conclude the following:

In 2004, the patient did not present structural brain disease (evaluated by clinical, imaging and neuropsychological examination). Between 2004-2008 there was an apparently healthy period.
In 2008, a dementia syndrome was reported, the value of which, in our view, is null and void, because good medical practice has not been complied with for a diagnosis of this kind and because "apparently" the patient has recovered from it. A hypothetical dementia syndrome was alleged 2 years after, although the patient remained about 1 year without clinical records.

It is during this period that the patient makes a will. That is, in the period after the doctor said to have improved and stabilized, despite the finding "depressed."

From the former, what can be safely stated is that the patient underwent long periods without medical consultation and that, even if he had any neurodegenerative pathology, it would be unstable in nature, recovering and worsening from time to time.

That is, in psychiatric terms, we have no way of saying that the person examined was completely healthy from the point of view of neurodegeneration (that is, that he did not have a dementia), as we have no means to say otherwise.

As stated in the introduction to this paper, it is not enough for someone to present a particular diagnosis (in this case, a diagnosis of dementia) in order to immediately deduce that this diagnosis would have implications for the ability to judge.

Even though we have seen that there are no means in the record to establish with certainty that at the time of the will, the person examined would have a diagnosis of dementia, let us do an academic exercise and consider that yes, he suffered from dementia at the time of the will.

What are the consequences of this putative diagnosis for the ability to judge and to want and to understand the scope of his acts, namely the will that he did?

If it were an Alzheimer's, Lewi-Bodies, or Frontotemporal disease, the consequences would be very dependent on the degree of dementia: grade 1 or mild (no attainment of the ability to judge); Grade 2 or moderate (partial attainment of the ability to judge); Grade 3 or severe (attainment of the ability to judge). That is, if we had a diagnosis of each of these types of dementia safely, we would have to determine the degree to consider a hypothetical alteration of the ability to judge that, even then, would have to be assessed by administering specific neuropsychological tests to determine (usually, when made according to the legis artis, they present a reliability rate in the order of $85 \%)$. Furthermore, any one of these types of dementias evolves inexorably into a worsening which, though variable from patient to patient, extends in the cognitive plane within 3-4 years.

On the contrary, if the patient had a putative diagnosis of a vascular dementia, which is caused by several small cerebral infarcts and usually installed in patients with cardiac pathology, characterized by the patients presenting in the early stages a better discernment than the patients with Alzheimer's type dementia, together with a relatively preserved personality for a longer period of time than the Alzheimer's type dementia. Symptoms may include periods of depression and mood swings, and the condition progresses with worsening (during small strokes) and periods of recovery and stabilization (after those small strokes). Usually people with this type of dementia die from cardiorespiratory arrest or stroke. In this type 
of dementia, the same question arises as to the relationship between dementia degrees and the ability to judge, as well as to instrumental measures for accurate assessment of the degree of attainment of the ability to judge, although it is possible to admit periods of stability and even improvement.

If a diagnosis could be made with the data we have, it would be vascular dementia and never Alzheimer's dementia. The reasons, albeit in terms of probability, but based on the medical events reported in the file, are as follows: (1) the patient presented periods of stabilization and compensation (although what the clinician meant by this designation is not known, (2) presented some periods of depression, which are also typical of vascular dementia; (3) dies, on 12 June 2013, for cardio-respiratory arrest, which is frequent in vascular dementia.

Conclusion: A- There are no reports in the records that allow us to affirm objectively that at the time of the will the patient suffered from changes in the capacity to judge, to want and to understand the scope of his act, or the opposite.

B- There is a suspicion that the patient is developing a dementia that, due to the evidence in the file, namely the fluctuations of his mental state, not only for the long periods without medical appointments, but also for the references, although vague, to periods of stability and compensation, as well as the cause of death, would most likely be a process of a vascular nature.

C- To have a neurodegenerative pathology - which could only be of the vascular type, for the reasons already mentioned - the probability of the patient being in a period of stability during the will would be very large for 3 order of reasons: (a) the nature of the insane process that has progressed throughout phases of worsening and recovery; (b) corroborated by the fact that the patient between September 2010 and June 2011 did not have any type of clinical record, it can be deduced that he did not need to be consulted, and that in the last pre-testament register (September 2010) the clinician reported that the patient was stable; (c) the fact that the patient suffers from cardiac pathology and died from cardio-respiratory arrest.

D - In view of the foregoing, we are of the opinion that at the time of the will, the probability of the patient having the capacity for judgment to want and to understand the scope of his act would be far superior to not being able to want and to understand the reach of the act.

\section{Conclusion}

One of the important aspects in this type of forensic psychiatric assessment is the analysis of the clinical course of the patient through clinical records or other information elements (such as family, friends, etc.) in order to establish three types of diagnosis: (1) the nosological diagnosis; (2) the diagnosis of the degrees of freedom and (3) the diagnosis of the causal relations between the mental state and the respective abilities of wanting within the time in which the act was made.

What the expert is therefore asked, in an assessment of this kind, is a statement on the presence or absence of a psychiatric disorder together with its severity, as well as a decision on the effects of that disorder on the possibilities of acting (or degrees of freedom) of the agent in question. When we refer to possibilities of acting we are referring to the possibilities of being able to decide in this or that way within the time in which the act was made. In fact, it is only with these three aspects that one can approximate the probability that, at the moment of the act, the patient would or would not be able to judge and evaluate the consequences of that act. Taken together, these considerations suggest that the assessment of the mental capacity involves a balanced judgment between the presence or absence of mental disorder, between the context of the will and the personality of the patient, even though the presence or absence of mental disorder predominates in this judgment.

In these two cases, the diagnostic discussion was fundamental for the elaboration of the $2^{\text {nd }}$ diagnosis - that of the degrees of freedom - in order to inform the court about the probability of the patient having the conditions of will and wanting need to validate the act in legal terms.

It is obvious that the necessary condition for the elaboration of the reports on forensic psychiatric autopsies is the existence of clinical records, without which these autopsies would not have been possible or, at most, would have led to spurious conclusions. Even so, as was emphasized in clinical cases, the diagnostic judgments were, at each one of the levels, elaborated in probabilistic form.

It is always up to the Court to supplement the data of a forensic psychiatric autopsy with other data (particularly witnesses) in order to prepare its decisions.

\section{References}

1. European Community Regulation (2012). EU Regulation $n^{\circ} 650 / 2012$

2. Marques-Teixeira J. Inimputabilidade e esquizofrenia na legislação portuguesa Inimputability and Schizophrenia in Portuguese Legislation. Saude Mental. 2008; 5: 7-11.

3. Radoilska L. Autonomy and mental disorder. Oxford: Oxford University Press; 2012.

4. Diário da República n 165/2015, Série 1 de 2015-08-25.

5. Stoljar N. Theories of autonomy. In Ashcroft, A. Dawson, H. Draper, JR. McMillan. Principles of health care ethics $2^{\text {nd }}$ ed. Chichester: John Wiley \& Sons Ltd; 2006. 11-17.

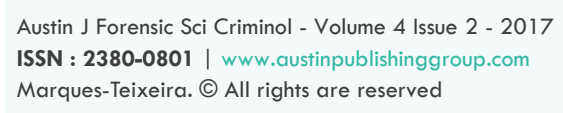

